\title{
Determination of ethanol concentration of ethanol/water mixture solutions with open ended coaxial method
}

\begin{abstract}
This study presents a simple and non-destructive procedure to determine ethanol concentration of ethanol/water solution at ambient temperature based on HP85070B open ended coaxial dielectric constant measurement. The motivation for this study stems from the fact that the 'true' concentration of ethanol in a 'labeled' container could be 'spoiled' due to hygroscopic nature of ethanol, evaporation and other factors. The dielectric constant measurements of eight samples with different molar fraction were validated with modified Cole-Cole-Debye (3CC) simulations. A 5th degree polynomial calibration equation was developed based on 3CC simulations with $\mathrm{R} 2=0.9998$ and used to estimate the 'true' concentration of three ethanol samples obtained from 'labeled' containers kept in laboratory. The ethanol concentrations of two of the samples were found to be much lower than what was indicated on their labels, hence, assumed spoiled.
\end{abstract}

Keyword: Ethanol concentration; Permittivity; Debye model; Open ended coaxial 